\title{
La infraestructura de datos espaciales del Gobierno de La Rioja (IDErioja): paradigma de una IDE
}

The spatial data infrastructure of the Regional Government of La Rioja, Spain (IDErioja): a paradigmatic SDI

\section{Ricardo CoRREDor FERnÁNDEZ (1), Gonzalo LóPEZ GARCíA (2)}

(1) Dirección General de las Tecnologías de la Información y la Comunicación del Gobierno de La Rioja, Avda. Lope de Vega, 43, 26006, Logroño, rcorredor@larioja.org. (2) Dirección General de Urbanismo y Vivienda del Gobierno de La Rioja, Marqués de Murrieta, 76, 26071, Logroño, gonzalo.lopez@larioja.org

\section{Resumen}

El Gobierno de La Rioja (España) inició en el año 2003 el denominado proyecto IDErioja, cuyo principal objetivo era desarrollar un sistema informático corporativo de información geográfica, soportado en bases de datos relacionales, que satisficiera los siguientes requisitos básicos: universalización del acceso a los datos geográficos, tanto para usuarios especializados como para los ciudadanos; centralización de la información geográfica en un único repositorio para aumentar la coherencia y seguridad de los datos; compatibilidad del dato geográfico con herramientas geomáticas de diferentes suministradores; integración de la información geográfica con los datos de aplicaciones de gestión administrativa; cumplir con los requisitos de la directiva europea INSPIRE y la norma ISO 19115 para la edición de metadatos; interoperabilidad con otras fuentes de datos geográficas de otros orígenes, mediante el uso de los servicios WMS de forma interactiva; y distribución de cartografía en tiempo real a través de un servidor Web Map Service (WMS), al que se puede acceder actualmente desde cualquier visualizador de mapas compatible con los estándares del Open Geoespatial Consortium (OGC).

Palabras clave: Iderioja. Infraestructuras de datos espaciales. Cartografía. WMS. La Rioja (España).

\section{Introducción}

El Gobierno de La Rioja (España) puso en marcha en el año 1989 un Sistema de Información Geográfica que ha ido creciendo y adaptándose al desarrollo tecnológico, hasta llegar a convertirse en un sistema corporativo.

Desde entonces el acceso a la información geográfica se ha realizado a través de la Intranet, estando restringida únicamente a los técnicos en gestión territorial, que además requerían para su manejo la utilización de programas informáticos de edición geográfica.

La posibilidad de incorporar datos espaciales en Sistemas de Gestión de Bases de Datos Relacionales (RDBMS) ha permitido abordar objeti-

\begin{abstract}
The Government of La Rioja (Spain) started in 2003 the IDErioja project, whose main objective was to develop a corporate computerized geographic information system, supported by relational databases, which satisfy the following basic requirements: universal access to geographic data, both for specialized users and citizens; centralization of geographic information in a single repository to increase data consistency and security; compatibility of geographic data with geomatic tools from different suppliers; integration of geographic information with administrative management applications data; complying with the requirements of European Directive INSPIRE and ISO 19115 for metadata edition; interoperability with other geographic data sources from other providers, using WMS services interactively; and real-time distribution of the cartography through a Web Map Service (WMS) server that can be accessed from any Open Geospatial Consortium (OGC) compatible map viewer.
\end{abstract}

Keywords: Iderioja. Spatial Data Infrastructures. Mapping. WMS. Rioja (Spain).

vos más ambiciosos, incrementando el aprovechamiento del dato espacial dentro de un ámbito corporativo.

Una vez analizadas las capacidades de los nuevos módulos espaciales de dichas bases de datos, el Gobierno de La Rioja, a través de la Dirección General de Urbanismo y Vivienda y la Dirección General de las Tecnologías de la Información y la Comunicación, abordó en el año $2003 / 2004$, el proyecto IDErioja, con la intención de poner la información geográfica al alcance de los usuarios, ciudadanos y procedimientos de gestión administrativa.

Para abordar este objetivo, ha sido fundamental el desarrollo tecnológico de lo que en el mundo 
de la información geográfica se conoce por Infraestructura de Datos Espaciales (IDE).

Una IDE, SDI en lengua inglesa, es un conjunto de protocolos y servicios orientados a la publicación y distribución de información geográfica a través de Internet.

Estos servicios, por medio de mecanismos cliente-servidor, atienden peticiones de distinta naturaleza, que ofrecen desde la remisión de una imagen cartográfica, hasta la descarga local de información espacial, o mecanismos sofisticados para búsqueda y localización de información basados en servicios de catálogo.

Por medio de estos mecanismos y estándares, de carácter internacional, se pretende definir un marco común con unas reglas fijas de comunicación entre usuarios y productores de información geográfica, con objeto de crear un gran mercado heterogéneo en donde sea posible descubrir y utilizar la información geográfica, sin importar en qué sistema o formato ésta ha sido generada.

El desarrollo a nivel nacional y europeo de las Infraestructuras de Datos Espaciales (IDE/SDI), la promulgación en el año 2007 de la Directiva Europea INSPIRE (Unión Europea, 2007), por la que se establece una infraestructura de información espacial en la Comunidad Europea, y el avance de los mecanismos de normalización ISO 19115 (International Organization for Standardization, 2003)—, se han integrado dentro del proyecto con objeto de cubrir tanto las necesidades internas como externas de información, de forma estándar y normalizada.

\section{Metas del proyecto}

Desde el diseño inicial y durante el proceso de construcción del proyecto IDErioja, se ha perseguido intensamente alcanzar los siguientes objetivos:

\subsection{Centralizar y compartir}

El Sistema de Información Geográfica utilizado hasta la fecha de puesta en marcha del nuevo proyecto en al año 2005 ha dado respuesta a las necesidades de un reducido colectivo de usuarios, que disponían de herramientas geomáticas especializadas, quedando fuera de este servicio las aplicaciones de gestión administrativa, al no ser éstas capaces de manejar los formatos de datos utilizados.

El nuevo modelo de datos, definido en el entorno de una base de datos relacional, universaliza el acceso a la información geográfica, al permitir a las aplicaciones de gestión adminis- trativa acceder sin restricciones a los datos espaciales.

La información geográfica se encuentra totalmente centralizada, pudiéndose descentralizar al mismo tiempo los trabajos orientados a su mantenimiento y actualización. Estas responsabilidades quedan así repartidas entre toda la organización, en función de las competencias de cada unidad.

Además de estos beneficios, el uso de una base de datos aumenta la coherencia de la información, permite un mayor control de la integridad referencial y mejora los aspectos de seguridad. El administrador del sistema asume la responsabilidad de realizar las copias de seguridad, evitar los accesos no permitidos, así como la gestión de las auditorias de acceso.

Con objeto de lograr la máxima interoperabilidad entre la base de datos y las herramientas de explotación, se ha definido un modelo de datos lo más neutro posible, intentando no condicionar éste a las exigencias de un software geográfico en particular.

Se ha de integrar la información geográfica en las aplicaciones de gestión administrativa como un atributo más, sin necesidad de alterar el modelo de datos de dichas aplicaciones.

\subsection{Normalizar}

El Comité Técnico 211 de la Organización Internacional para la Estandarización (ISO), se encuentra todavía en un proceso de definición de la serie de normas 19100 , orientadas a la estandarización de múltiples aspectos relativos a al tratamiento y gestión de la información geográfica digital.

La existencia de una normativa ISO, una vez que ésta sea asumida por el Comité Europeo de Normalización (CEN) y por la Asociación Española de Normalización y Certificación (AENOR), aporta seguridad en el desarrollo y puesta en marcha de los modelos de datos geográficos, lo que finalmente redunda en una mejora de la calidad y en el adecuado aprovechamiento de los datos. En el diseño del modelo se han tenido en cuenta aspectos conceptuales ya definidos en la normativa ISO. EI tratamiento de metadatos (datos sobre los datos) se ha implementado en Oracle, desarrollando para ello una aplicación en Internet, según lo especificado en la norma ISO 19115 y lo establecido para el Núcleo Español de Metadatos (NEM) por la Comisión Especializada de la Infraestructura de Datos Espaciales del Consejo Superior Geográfico. 
Otro aspecto de la estandarización que se ha tenido en cuenta ha sido lo especificado en las reglas de implementación (implementation rules, IR) desarrolladas en el marco de la directiva europea.

Por otra parte, el Consejo Superior Geográfico (CSG), a través de la Comisión Especializada de Normas Geográficas (CENG) ha definido la estructura de la Base Topográfica Armonizada 1:5000/1:10.000 (BTA) (Consejo Superior Geográfico, 2010), como solución para la armonización de las bases topográficas regionales. El proyecto IDErioja también incorporará dicha norma en su desarrollo.

\subsection{Distribuir}

Todo sistema de información que se precie, debe arbitrar mecanismos de distribución de la información que puedan atender las necesidades de cualquier usuario, en cualquier entorno.

El proyecto IDErioja ha tenido en cuenta el estándar WMS definido por el Open Geospatial Consortium (Open Geospatial Consortium, 1994-2013), sobre el que se apoya el desarrollo de las Infraestructuras de Datos Espaciales, para ofrecer en tiempo real acceso a la información geográfica de que dispone. La consulta de los usuarios a la información de la base de datos se realiza a través de un servidor OGCWMS al que se puede acceder desde cualquier visualizador de mapas compatible OGC.

Con objeto de ofrecer al usuario que accede a través de nuestras páginas, el mayor nivel de servicio, el Gobierno de La Rioja ha desarrollado visualizadores geográficos propios basados en sofware libre que permiten la consulta de la información geográfica y alfanumérica de la base de datos.

Intentando llevar el objetivo de distribuir la información a su cota máxima, en este mismo visualizador, se puede presentar la información geográfica procedente de la administración local, al estar la información topográfica urbana de cada municipio accesible a través de los servicios WMS, que con este objetivo se han desarrollado para la práctica totalidad de los municipios de La Rioja. El usuario puede tener así una visión completa de todas las fuentes de datos espaciales de la administración regional y local de la Comunidad Autónoma de La Rioja.

\section{Solución implantada}

En la solución adoptada, hay que subrayar que todas los datos, tanto los referentes a la información geográfica (vectorial), como a la información alfanumérica y todos los relacionados con la administración del sistema, se encuentran almacenados en un único repositorio, que en nuestro caso es una única base de datos Oracle con la extensión Spatial.

Todos los objetos lógicos de información (tablas) se han diseñado de acuerdo con las formas normales, utilizadas habitualmente en el modelado de bases de datos relacionales. Adicionalmente, por cuestiones de eficacia y rendimiento, la información relativa a las imágenes ráster (ortofotos, mosaicos -tiles-, relieves, mapas generales, etc.) se ubican en un servidor de datos adicional.

Todo el software de IDErioja, se ha diseñado para utilizarse íntegramente desde Internet y consta de las cuatro grandes áreas, que se describen en los siguientes apartados.

\subsection{GeoPortal www.iderioja.org}

A partir de esta página se puede acceder a todos y cada uno de los módulos de los que se compone el sistema. Cabe destacar la posibilidad de descargas de datos geográficos y la suscripción al canal RSS de IDErioja. Algunas de estas áreas, son de uso interno del Gobierno de La Rioja, por lo que el usuario debe autentificarse para acceder a las mismas.

\subsection{Visualizador regional}

El visor de mapas constituye la ventana de presentación en Internet de la cartografía regional. Permite la consulta interactiva a través de Internet, de toda la información geográfica, es decir, la vectorial de la base de datos, la ráster del servidor de mosaicos de imágenes y la disponible en otros servidores WMS, para representarla de forma integrada en un único mapa (Figura 1). Las funcionalidades que proporciona son:

- Leyenda de las capas con posibilidad de ordenación, ocultación, indicación de capa activa para ver sus datos, niveles de transparencia y acceso a metadatos.

- Acceso a capas provenientes de otros servidores OGC-WMS.

- Mapa guía para facilitar localizaciones y desplazamientos.

- Herramientas de navegación: Acceso directo a municipios, zoom, centrado del mapa, medición de distancias y áreas.

- Inclusión de anotaciones en el mapa (textos, símbolos, puntos, líneas y polígonos).

- Acceso a Street View de Google Maps y Panoramio de Google Earth. 
Paneles informativos inferiores: coordenadas y barra de escala.

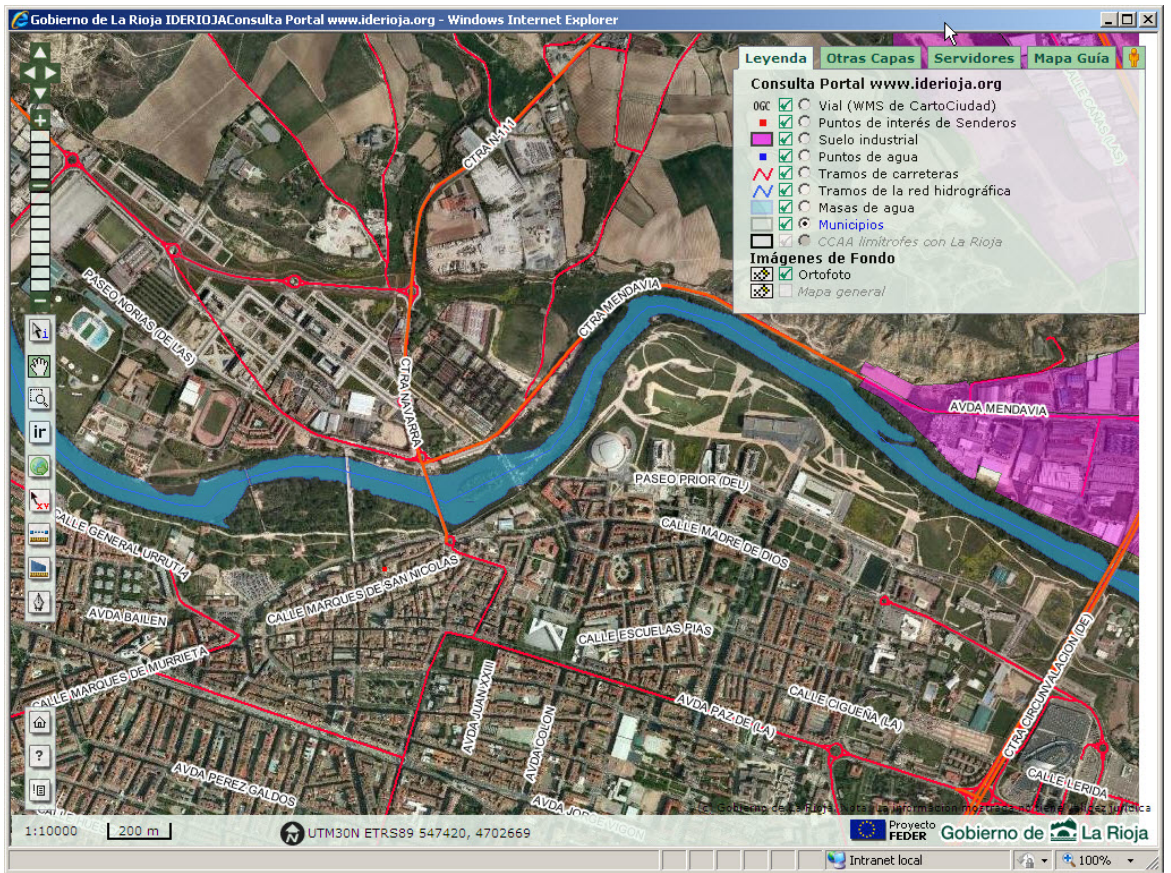

Figura 1. Visualizador regional

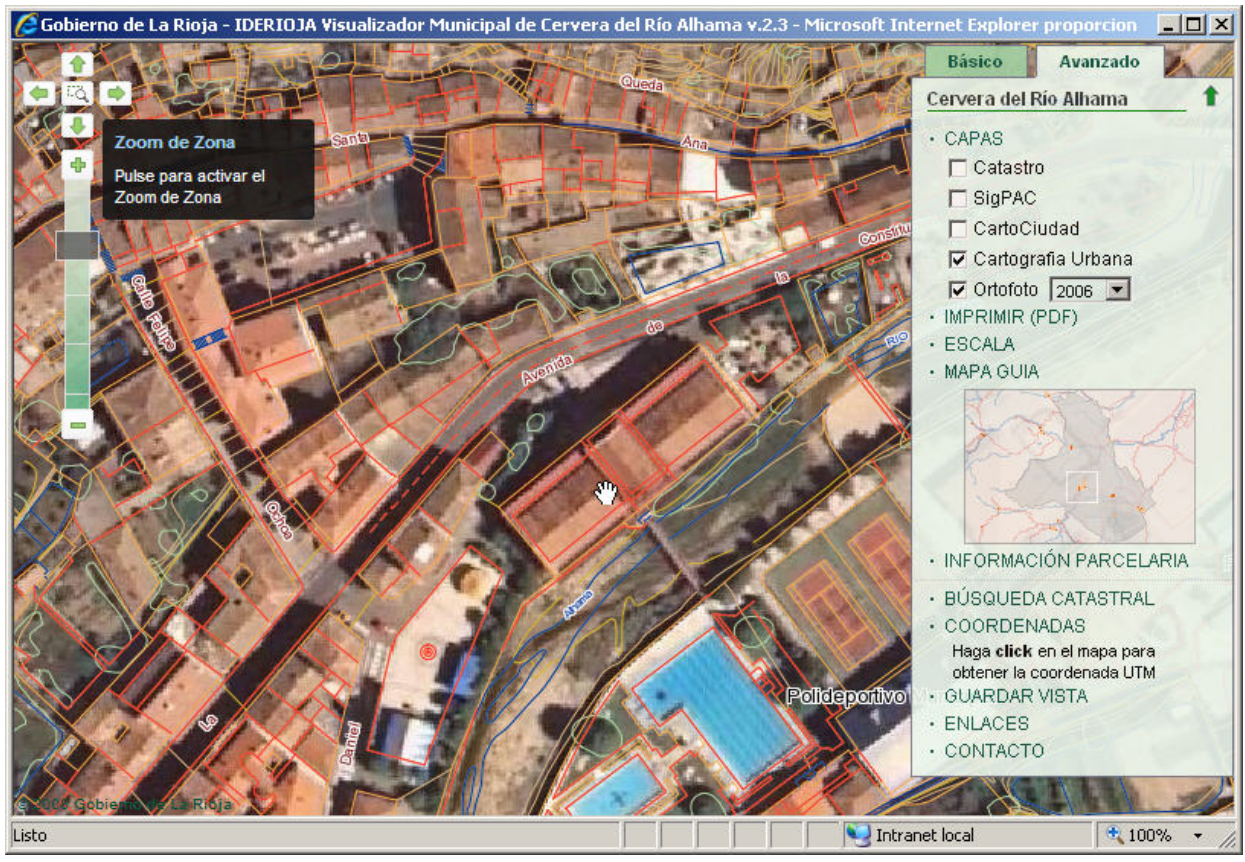

Figura 2. Visualizadores municipales

\subsection{Visualizadores municipales}

Con la finalidad de tener una infraestructura completa de datos espaciales del territorio de La Rioja, se ha incorporando la cartografía urbana de 172 de los 174 municipios existentes en la Comunidad Autónoma de La Rioja. Esta información geográfica, por motivos de economía de recursos, no se encuentra ubicada en la base de datos corporativa, sino en ficheros con formato shapefile.

Aplicando tecnologías de software libre y sirviéndonos se de la conectividad proporcionada por los servidores OGC-WMS, se ha construido 
un visualizador geográfico, personalizado para cada entorno municipal, así como un servidor (MapServer), que presenta gráficamente la información de cada municipio además de atender peticiones de información geográfica provenientes de otros entornos.

El acceso siempre es libre, sin necesidad de registrarse en el sistema y estando diseñada para que el acceso sea través de la página institucional de cada municipio, ya que el desarrollo se ha adaptado geográficamente para cada uno de los municipios de La Rioja (Figura 2, en la página anterior).

\subsection{Aplicación de gestión}

La aplicación de gestión es el entorno en el que se administra, mantiene y consulta toda la infraestructura de datos espaciales y a la que pueden acceder tanto usuarios anónimos, a los que se les muestra la información pública ("Zona pública"), como usuarios registrados, cuyo espectro de actuación va desde el mantenedor de una capa al administrador general de IDErioja.

En los siguientes subapartados se describen los módulos que componen toda la aplicación. Dependiendo de las autorizaciones de cada usuario, éste podrá acceder a todas ellas o a un subconjunto de funciones de la aplicación, se- gún una configuración que se puede adaptar a las necesidades y preferencias de cada usuario.

\subsubsection{Usuarios y funciones de menú}

Permite el mantenimiento de usuarios registrados en el sistema y la personalización de sus menús en base a un sistema de autorizaciones basado en áreas, funciones y grupos.

\subsubsection{Catálogo de capas}

Definición de todos los objetos de la base de datos, con información cartográfica, es decir, que contengan la geometría expresada en coordenadas UTM ETRS89, clasificadas según la estructura organizativa de familia, subfamilia y tema, y su relación con los metadatos ISO 19115.

\subsubsection{Configuración del visualizador regional}

El visualizador regional, se ha diseñado para que pueda configurarse mediante una serie de parámetros que ajustan su respuesta gráfica ,m tales como el sistema de coordenadas en que se va a representar la información geográfica o la capa de rango máximo que va a delimitar el entorno territorial. Esta peculiaridad permite que el visualizador pueda ser utilizado de forma universal por todas las aplicaciones del sistema.

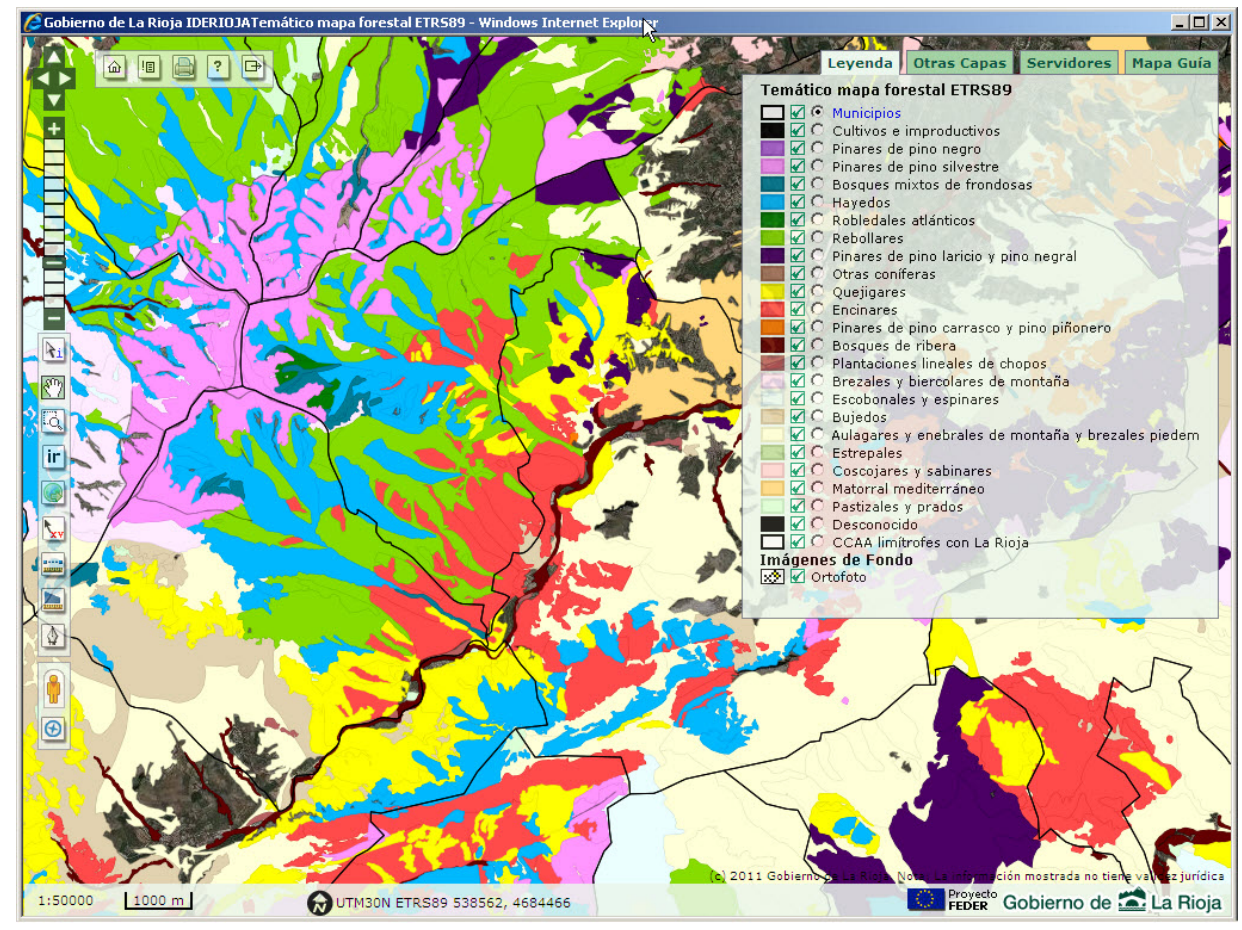

Figura 3. Mapas temáticos

También se definen las capas y los datos alfanuméricos que se desea representar, indicando los estilos de representación de las mismas. Se posibilita la definición de datos no existentes en 
la propia capa y que son el resultado del acceso a otras tablas o de cálculo, como por ejemplo, el área o perímetro de la geometría de la capa.

Dado que el visualizador puede acceder a cualquier servidor OGC-WMS de la red, éstos se pueden incorporar al mismo, bien sea a partir de una lista de servicios, como de forma interactiva a voluntad del usuario que realiza la consulta al visualizador.

Asimismo se pueden modelar mapas temáticos en función de los valores de algún atributo de la capa (Figura 3).

\subsubsection{Cartografía.}

Este módulo facilita el acceso toda la información geográfica con datos de sus geometrías (puntos, líneas o polígonos), datos alfanuméricos y las tablas de códigos asociadas. Se compone de:

- Capas temáticas organizadas según la directiva INSPIRE.
- Base Topográfica Armonizada, BTA 1:5000 y $1: 10.000$

- Encuesta de infraestructura y equipamientos locales.

- Sistema de Información Urbanística.

Toda la información alfanumérica y espacial de las capas geográficas se puede mantener con las herramientas que aporta el sistema, sin necesidad de recurrir a otras aplicaciones geomáticas (Figura 4).

La edición de las geometrías está también habilitada para GeoMedia Pro (Intergraph), siendo posible mantener la información vectorial y alfanumérica de cada capa. En cualquier caso los datos geográficos y alfanuméricos se validan utilizando las propiedades topológicas y de integridad referencial de Oracle Spatial. En modo consulta, también se pueden utilizar herramientas de software libre como gvSIG, Quantum GIS y el software propietario desarrollado por la empresa ESRI.

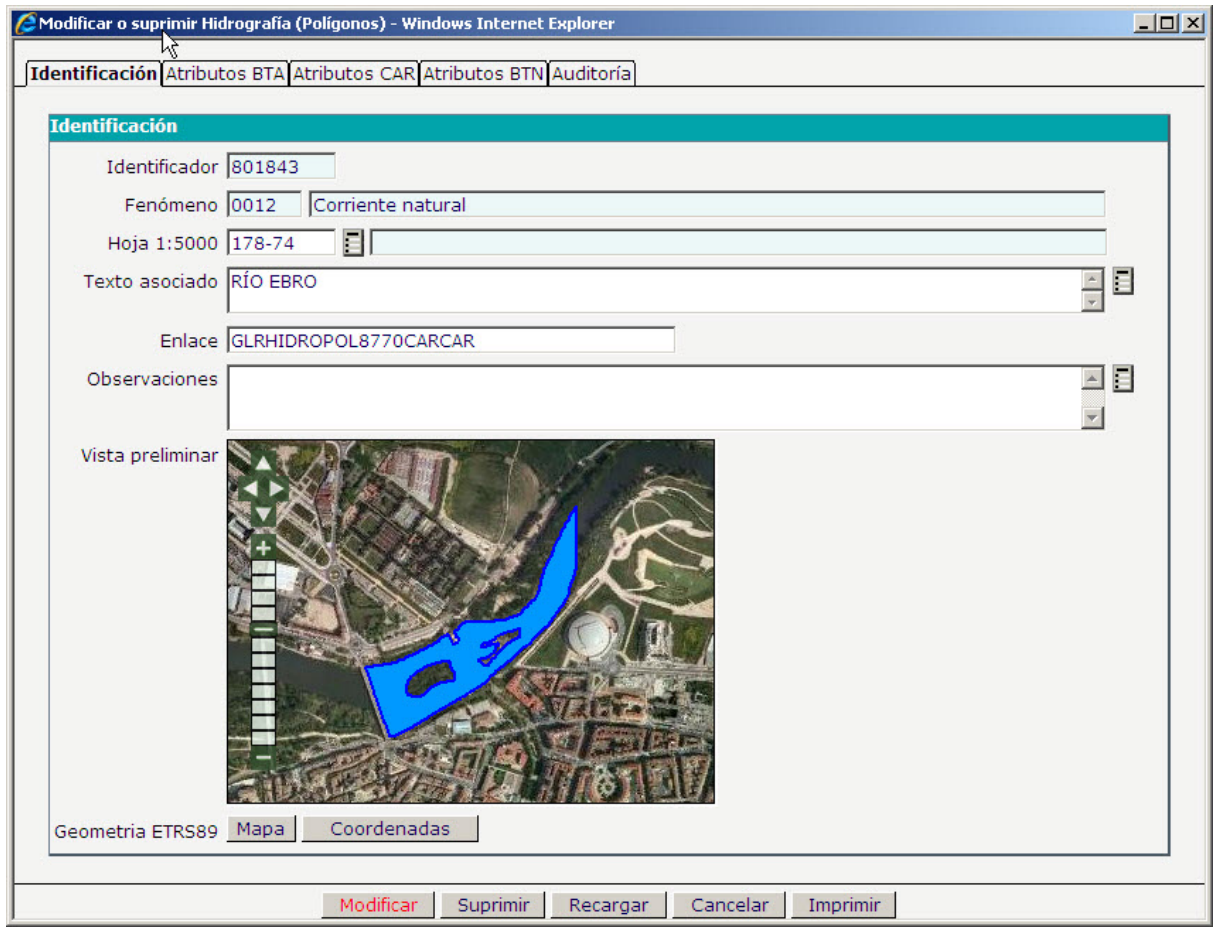

Figura 4. Ventana de datos de un elemento

Las herramientas anteriores (clientes pesados) utilizan tecnología cliente/servidor, lo que requiere la instalación de licencias en cada ordenador personal; recientemente se ha desarrollado un GeoEditor que, en el entorno de visualizador regional, permite la edición geográfica desde Internet, sin licencias de usuario.

\subsubsection{Modelado de capas (Geomatic Layers Engine)}

El modelado de capas es una herramienta de reciente incorporación, que permite la generación de capas geográficas sin la intervención de profesionales informáticos. 
Los usuarios especializados definen cada capa con sus atributos asociados, sobre los que se realizan las validaciones propias de la naturaleza de los mismos (numéricos, códigos de listas de valores, ...) y otras definidas por los usuarios, como la obligatoriedad o no duplicidad.

Se autoriza la incorporación y modificación de atributos aún con datos existentes en las tablas.

Aunque se ha diseñado para la agregación de nuevas capas temáticas, también se utilizará para la incorporación de toponimia, imágenes y cartoteca, gracias a las utilidades de carga y representación de imágenes.

Los datos completos de una capa se pueden actualizar con diferentes procedimientos:

- A través de las herramientas geomáticas GeoMedia Pro o FME, que pueden mantener datos geográficos y alfanuméricos.

- Con la aplicación de gestión para el mantenimiento de los datos alfanuméricos y la incorporación de imágenes (como cualquier otra capa del sistema), ya que se genera $H T M L$ y javascript de manera dinámica, en función de la definición de cada capa.

- Mediante el GeoEditor, un editor de cartografía en Internet que se invoca desde la aplicación java y con el que, de igual manera que con las herramientas geomáticas, se pueden mantener datos geográficos y alfanuméricos.

\subsubsection{Metadatos ISO 19115, perfil CAR}

A través de la aplicación web es posible mantener en Oracle el catálogo de metadatos de la información geográfica existente.

El diseño está basado en el núcleo de la norma internacional ISO/TC 211 DIS 19115, en lo definido para el Núcleo Español de Metadatos (NEM versión 1.0) y en las agregaciones particulares de la Sección de Sistemas de Información Geográfica y Cartografía de la Consejería de Obras Públicas, Política Local y Territorial.

Dada la complejidad del modelo de metadatos, el mantenimiento de los distintos apartados se ha facilitado mediante una pantalla que muestra en forma de árbol la estructura y el estado de la información mediante la utilización de iconos cromáticos.

También se permite la exportación de toda la información de cada metadato al formato estructurado XML.

\subsubsection{Utilidades}

Este módulo engloba funciones propias de la administración del sistema y tiene como objetivo el que no sea necesario utilizar herramientas de otros proveedores:

- Configuración del GeoPortal IDErioja, incluyendo la gestión de las noticias y los canales RSS.

- Asignación de autorizaciones a los usuarios de herramientas geomáticas, para asignar los permisos de acceso sobre objetos de la base de datos Oracle, para la edición de las capas cartográficas.

- Herramientas de análisis de geometrías e índices espaciales, específicas de Oracle Spatial.

- Mantenimiento de parámetros de uso general en el sistema, por ejemplo, las coordenadas $x$ e y máxima y mínima, del territorio de la Comunidad Autónoma.

- Consulta de las auditoría de los objetos que contienen información de carácter personal.

\subsubsection{Monitor Oracle}

Para facilitar a los diseñadores y administradores del proyecto la comprensión del sistema de almacenamiento de los objetos espaciales, se han desarrollado un conjunto de funciones que sirven para monitorizar y controlar el núcleo de la base de datos en la que se apoya toda la cartografía vectorial: diccionario de objetos, sesiones activas, almacenamiento, estadísticas, etc.

Se pretende así hacer más transparente la base de datos Oracle a técnicos profanos en dicho entorno.

También incluye la visualización del middleware utilizado con las herramientas geomáticas, es decir, los módulos intermedios que actúan como conductores entre Oracle, GeoMedia, ArcSDE o FME.

\section{Plataforma tecnológica}

La arquitectura del sistema esta construida para dar cumplimiento a los objetivos del sistema, a los estándares del Gobierno de La Rioja y las tendencias actuales del software libre; y consta de los siguientes elementos:

- Base de datos Oracle. Se hospeda en un servidor corporativo que contiene una única base de datos geográfica con la extensión Spatial, con todas las entidades con información vectorial. Esta base de datos tiene los 
enlaces necesarios con las bases de datos de gestión, para compartir la información geográfica con las aplicaciones administrativas.

- Servidor de aplicaciones web. La aplicación de gestión, que va a administrar todo el sistema y va a permitir el acceso a todos los datos geográficos, está desarrollada con Java estándar J2EE. Para la explotación de este componente del proyecto, se utiliza software libre, en concreto, Apache-Tomcat, bajo el sistema operativo LINUX-CentOS.

- Servidores de mapas web. El visualizador regional y los visualizadores municipales están construidos con software libre, en concreto OpenLayers. Para la generación de las imágenes de los mapas desde la base de datos se emplea Minnesota MapServer, que también es software libre.

- Servidores OGC. Para la publicación de los diferentes servidores WMS, WFS y WCS de IDErioja (Capas públicas, Cartografía municipal, Base topográfica armonizada, etc.) se utiliza también Minnesota MapServer, para lo que se ha preparado un programa que genera cada servidor de forma automática.

- Servidor ArcSDE. Este servidor se utiliza como conector de Oracle Spatial con todas las herramientas geomáticas de ESRI $\left(\operatorname{Arc}^{*}\right)$.

- Herramientas geomáticas. El sistema está preparado para la edición de cartografía con GeoMedia PRO de Intergraph. También puede actualizarse la información alfanumérica y geométrica a través de procesos diseñados con FME (Feature Manipulation Engine). En modo consulta, se puede además acceder con las herramientas de ESRI, con software libre como gvSIG y, en general, con todas las herramientas que entiendan el formato del dato geográfico de Oracle Spatial.

- Navegadores de usuario. El acceso al sistema se puede realizar desde los navegadores Internet Explorer, Mozilla Firefox o Google Chrome; y en ningún caso se requiere la descarga de componentes adicionales.

\section{IDErioja en la IDE española y europea}

IDERioja, es un proyecto orientado a la diseminación de datos espaciales, que como tal se entronca dentro de lo que se denomina la Infraestructura de Datos Espaciales Española cuyos objetivos y desarrollo se puede consultar en la página web http://www.idee.es-, constituyendo ésta un nodo más del entramado europeo y global de datos espaciales, que la Directiva INSPIRE trata de normalizar.

La singularidad del proyecto IDErioja estriba en el desarrollo de herramientas web para el mantenimiento de la información, sin estar sometido al dictado de un entorno o herramienta geomática específica; y en la posibilidad que ofrece de extender la utilidad de la IDE al conjunto de aplicaciones administrativas de carácter general, convirtiéndolas de esta manera en centros productores de datos geográficos.

Existen en todas las regiones de España distintas iniciativas con los mismos o parecidos objetivos, entre las que cabe destacar la IDE de Navarra (IDENA) (Infraestructura de Datos Espaciales de Navarra, 2012) o la desarrollada por la Generalitat de Cataluña (IDEC) (Institut Cartogràfic de Catalunya, 2006-2013), además de los servicios IDE ofrecidos a nivel estatal por el Instituto Geográfico Nacional (Consejo Superior Goegráfico, 2007-).

\section{Conclusiones}

Consideramos a IDErioja un auténtico paradigma de cómo deben diseñarse las IDEs.

El modelo es totalmente transferible a cualquier otra administración pública que deba manejar información geográfica, ya que entre otros, cumple con los requisitos de la directiva INSPI$\mathrm{RE}$; está implementado cumpliendo los estándares internacionales referidos a este campo (ISO 19115); y ha adoptado el nuevo modelo de datos de la Base Topográfica Armonizada 1:5000 (BTA), que ha sido aprobada formalmente la por la Comisión Permanente del Consejo Superior Geográfico. Se prevé una paulatina adopción de dicho modelo de datos por las organizaciones cartográficas españolas, ya que de esta manera se va a permitir la interoperabilidad de la información geográfica digital de la cartografía topográfica generada por la administración, tanto a nivel nacional como regional y local.

Además soluciona una problemática común a todas las administraciones, que es la gestión centralizada de la información geográfica, su integración en los procesos administrativos y su distribución pública.

\section{Referencias}

Consejo Superior Geográfico (2010). Comisión Especializada de Normas Geográficas: Base Topográfica Armonizada.Madrid: Consejo Superior Geográfico, 2010. http://www.csg-cnc.es/web/cnccontent/bta.html.

Consejo Superior Goegráfico (2007-). Geoportal IDEE. Madrid: Ministerio de Fomento, Consejo Superior Geográfico, 2007-. http://www.idee.es/web/guest/directoriode-servicios 
Infraestructura de Datos Espaciales de Navarra (2012). IDENA. Pamplona: Gobierno de Navarra, 2012. http://idena.navarra.es.

Institut Cartogràfic de Catalunya (2006-). Geoportal IDEC. Barcelona: Institut Cartogràfic de Catalunya. http:// www.geoportal-idec.cat.

International Organization for Standardization (2003). ISO 19115: 2003 Geographic information Metadata. Genève: ISO, 2003.
Open Geospatial Consortium (1994-2013). OGC Open Geospatial Consortium. http://www.opengeospatial.org/.

Unión Europea (2007). Directiva 2007/2/CE del Parlamento Europeo y del Consejo, de 14 de marzo de 2007, por la que se establece una infraestructura de información espacial en la Comunidad Europea (Inspire). // Diario Oficial de la Unión Europea. L108 (25-4-2007) 1-14.

Fecha de envío: 2012-05-15.

Fecha de aceptación: 2012-08-21. 
